\title{
Incidence and Mortality of Treated Primary Sjögren's Syndrome in Taiwan: A Population-based Study
}

\author{
MENG-YU WENG, YU-TUNG HUANG, MING-FEI LIU, and TSUNG-HSUEH LU
}

\begin{abstract}
Objective. To estimate the incidence and mortality of treated primary Sjögren's syndrome (pSS) by sex and age group in Taiwan.

Methods. We used claims data of the Bureau of National Health Insurance (NHI) of Taiwan from 2005 to 2007 for analysis. According to the NHI, pSS is classified as one of the financially catastrophic illnesses and patients with pSS could be exempted from copayment of all medical costs. To obtain a catastrophic illness certificate (CIC) for pSS, patients are required to meet the criteria of the American-European Consensus Group for pSS, and are reviewed by a committee. Patients approved for receipt of a CIC for pSS for the first time were defined as incident cases of treated pSS.

Results. A total of 3352 incident cases occurred between 2005 and 2007. The estimated mean annual incidence was 6.0 per 100,000 inhabitants (95\% CI 5.8-6.2) for both sexes, 11.0 (95\% CI 10.6-11.4) for women and 1.1 (95\% CI 1.0-1.2) for men, with a female/male ratio of 9.9 (95\% CI 8.8-11.1). Incidence increased with age, peaking at age 55-64 years in women and 65-74 years in men. The mortality rate was 33.4 per 1000 case person-years for men and 11.4 for women, with a male/female rate ratio of 2.9 (95\% CI 1.7-5.3).

Conclusion. The incidence of treated pSS in women is 10 times that in men. Nevertheless, pSS mortality in men is 3 times that in women. (First Release Jan 15 2011; J Rheumatol 2011;38:706-8; doi:10.3899/jrheum.100883)
\end{abstract}

\section{Key Indexing Terms:}

\section{SJÖGREN'S SYNDROME INCIDENCE MORTALITY EPIDEMIOLOGY TAIWAN}

Primary Sjögren's syndrome (pSS) is an autoimmune disorder characterized by dry eyes and a dry mouth. Estimation of the incidence and mortality of pSS is an essential step in assessing the clinical course and disease burden. However, few population-based epidemiological studies have examined the incidence and mortality of pSS. The incidence of physician-diagnosed pSS has been estimated as 3.9 per 100,000 inhabitants in Ljubljana, Slovenia, 3.9 in Olmsted County, Minnesota, USA, and 5.3 in the northwest region of Greece $^{1,2,3}$. Mortality studies suggest that the incidence of mortality in patients with pSS is not significantly higher than that in the general population ${ }^{3,4,5,6,7}$. One limitation of previous studies has been confinement to a specific geographic region, with a small number of cases, which did not enable further analysis of the incidence and mortality of pSS by sex and age group. Therefore, using claims data of the National Health Insurance (NHI) service of Taiwan, our

From the Section of Allergy, Immunology, and Rheumatology, Department of Internal Medicine, National Cheng Kung University (NCKU) Medical College and Hospital; NCKU Research Center for Health Data and Institute of Public Health, College of Medicine, National Cheng Kung University, Tainan; and Department of Gerontological Care and Management, Chang Gung Institute of Technology, Taoyuan, Taiwan.

M.Y. Weng, MD, MS, Rheumatologist; Y.T. Huang, PhD, Lecturer; M.F. Liu, MD, Section Head; T.H. Lu, MD, PhD, Associate Professor. Address correspondence to Dr. T.H. Lu, Institute of Public Health, College of Medicine, National Cheng Kung University, No. 1, Dah Hsueh Road, Tainan 701, Taiwan.E-mail: robertlu@mail.ncku.edu.tw

Accepted for publication November 15, 2010. study aimed to determine the incidence and mortality of treated pSS by sex and age group.

\section{MATERIALS AND METHODS}

Claims data of the NHI of Taiwan were used for estimation purposes. The NHI program is a mandatory, compulsory, single-payer social health insurance system in which all citizens are obliged to participate. Implemented in 1995, NHI covered $99.5 \%$ of the population of Taiwan ( 23 million) by the end of $2008^{8}$. The NHI Research Database has been released to researchers in an electronically encrypted form since 1999; this includes records of all medical benefit claims for ambulant care and inpatient care and is used extensively in epidemiological studies.

To avoid financial hardship for families coping with major injuries/illnesses, the NHI specifies 31 categories of catastrophic illness (e.g., cancers, hemophilia, autoimmune diseases, chronic renal failure, etc.) that are exempt from copayment of all medical costs, and the attending physician of any patient diagnosed as falling into one of these categories of catastrophic illness under the Department of Health guidelines can submit related information to complete an application for a catastrophic illness certificate (CIC). Applications are formally reviewed by a committee, and if approved, patients are exempted from copayment of all medical costs 9 .

Most autoimmune diseases are qualified as catastrophic illnesses by the $\mathrm{NHI}$ and $\mathrm{pSS}$ is one of them. To obtain a CIC for pSS, the attending physician of a patient is required to provide relevant clinical and laboratory information as part of the application for review, and the review committee will assess applications according to the criteria of the American-European Consensus Group for $\mathrm{pSS}^{10}$. The NHI began to reimburse medication fees for some drugs used to treat pSS (e.g., pilocarpine) in 2004, and the number of approved CIC applications for pSS has increased and remained stable since 2005 . We thus confined our analysis to the years 2005 through 2007.

Patients approved for receipt of a CIC for pSS for the first time were

Personal non-commercial use only. The Journal of Rheumatology Copyright $($ C 2011. All rights reserved. 
defined as incident cases of treated pSS. We excluded patients with both a CIC for pSS and a CIC for any other autoimmune disease, such as systemic lupus erythematosus, rheumatoid arthritis, other connective tissue diseases, etc., who should instead be classified as having secondary SS. There is an item within the catastrophic illness records to denote whether a patient has died, and we were thus able to ascertain the number of deaths among patients in receipt of a CIC for pSS during the study period.

We calculated sex-age-specific and age-adjusted incidence of treated pSS as the number of incident cases per 100,000 inhabitants. The age distribution of standard population (i.e., population of Taiwan for year 2006) is given in Table 1 . The mortality of treated pSS was computed as the number of incident cases in which death occurred per 1000 case person-years by sex and age group. We also calculated the standardized mortality ratio (SMR) of patients with treated pSS as compared with the general population of Taiwan matched by sex, age (ranges 0-24, 25-44, 45-64, 65-74, and $\geq 75 \mathrm{yrs}$ ), and calendar years (2005-2007). The $95 \%$ confidence intervals of SMR were estimated using the Poisson distribution.

\section{RESULTS}

Incidence. A total of 3352 patients aged $\geq 15$ years were approved for receipt of a CIC for pSS for the first time between 2005 and 2007. The mean age of treated pSS patients was 53 years (SD 14) for women and 58 years (SD $15)$ for men, a significant statistical difference ( $p<0.001$, $\mathrm{t}$ test). The age-adjusted mean annual incidence was 6.0 per 100,000 inhabitants (95\% CI 5.8-6.2) for both sexes, 11.0 (95\% CI 10.6-11.4) for women and 1.1 (95\% CI 1.0-1.2) for men, with a female/male ratio of 9.9 (95\% CI 8.8-11.1). The incidence of pSS increased with age, peaking at 55-64 years in women and 65-74 years in men. The female/male ratio was the largest in the 35-54 year age group (Table 2). Mortality. The mortality rate was 13.4 per 1000 case person-years (95\% CI 10.2-16.7) for both sexes, 33.4 (95\% CI 16.5-50.4) for men and 11.4 (95\% CI 8.2-14.6) for women,

Table 1. Age distribution of the general population in Taiwan, 2006.

\begin{tabular}{lccc}
\hline Age, yrs & All & Women & Men \\
\hline $0-24$ & $7,615,270$ & $3,662,509$ & $3,952,762$ \\
$25-44$ & $7,594,956$ & $3,756,639$ & $3,838,318$ \\
$45-64$ & $5,363,118$ & $2,694,461$ & $2,668,658$ \\
$65-74$ & $1,313,230$ & 682,896 & 630,333 \\
$75+$ & 936,975 & 450,605 & 486,371 \\
Total & $22,823,550$ & $11,247,109$ & $11,576,441$ \\
\hline
\end{tabular}

with a male/female mortality rate ratio of 2.9 (95\% CI 1.7-5.3). For both sexes, mortality increased with age (Table 3). The SMR of patients with treated pSS was 1.12 (95\% CI 0.86-1.43) for both sexes, 1.30 (95\% CI 0.96-1.72) for women and 1.54 (95\% CI 0.86-2.53) for men, compared with the general population.

\section{DISCUSSION}

Our findings suggest that the incidence of treated pSS was 10 times higher in women than in men, and the difference in incidence between sexes was greatest in the middle-aged group. Despite the lower incidence in men, mortality was 3 times higher in men than in women. The mortality rate for patients with pSS of either sex was not significantly higher than that of the general population of Taiwan.

One of the strengths of this study was that it was the first nationwide population-based epidemiological study of pSS, and the large number of incident cases allowed us to calculate the incidence and mortality by sex and age group. The second strength of the study was the use of a strict definition of incident cases, as all patients approved for receipt of a $\mathrm{CIC}$ for pSS are required to meet the criteria of the American-European Consensus Group for pSS as reviewed by a committee.

One limitation of using catastrophic illness records to define pSS cases was that not all patients with physician-diagnosed pSS applied for a CIC for pSS. Some patients with pSS who did not experience a financial burden in copayment of medications might not apply for a CIC for $\mathrm{pSS}$, which would result in underestimation of the incidence rate. However, by contrast, some old prevalent cases might also have been misclassified as incident cases, which result in overestimation of the incidence rate. As the NHI began to reimburse the medication fees for some drugs used to treat pSS in 2004, some patients diagnosed with pSS before 2004 might have applied for a CIC for pSS in 2005. We believe this is one explanation why the incidence rate estimated in this study was higher than in previous studies ${ }^{1,2,3}$. It is reasonable that overestimation would be more likely to occur among elderly persons who were more vulnerable to financial burden for copayment of medications. Further studies are needed to clarify the possible effects of misclassification.

Table 2. Age-specific and age-adjusted incidence rate (per 100,000 inhabitants) of treated primary Sjögren's syndrome in Taiwan, $2005-2007$.

\begin{tabular}{|c|c|c|c|c|c|c|c|}
\hline \multirow[b]{2}{*}{ Age, yrs } & \multicolumn{2}{|c|}{ Total } & \multicolumn{2}{|r|}{ Female (F) } & \multicolumn{2}{|c|}{ Male (M) } & \multirow[b]{2}{*}{ F/M Ratio $(95 \% \mathrm{CI})$} \\
\hline & No. & Rate $(95 \% \mathrm{CI})$ & No. & Rate $(95 \% \mathrm{CI})$ & No. & Rate $(95 \% \mathrm{CI})$ & \\
\hline $15-24$ & 61 & $0.6(0.5-0.8)$ & 56 & $1.1(0.8-1.4)$ & 5 & $0.1(0.0-0.2)$ & $11.9(4.8-29.8)$ \\
\hline $25-34$ & 273 & $2.4(2.1-2.7)$ & 251 & $4.4(3.9-5.0)$ & 22 & $0.4(0.2-0.5)$ & $11.7(7.6-18.1)$ \\
\hline $35-44$ & 538 & $4.7(4.3-5.1)$ & 502 & $8.9(8.2-9.7)$ & 36 & $0.6(0.4-0.8)$ & $14.2(10.1-19.9)$ \\
\hline $45-54$ & 938 & $9.1(8.5-9.7)$ & 877 & $17.0(15.9-18.1)$ & 61 & $1.2(0.9-1.5)$ & $14.4(11.1-18.7)$ \\
\hline $55-64$ & 752 & $13.0(12.1-14.0)$ & 684 & $23.4(21.6-25.1)$ & 68 & $2.4(1.8-3.0)$ & $9.7(7.6-12.5)$ \\
\hline $65-74$ & 533 & $13.5(12.4-14.7)$ & 457 & $22.3(20.3-24.4)$ & 76 & $4.0(3.1-4.9)$ & $5.6(4.4-7.1)$ \\
\hline $75+$ & 257 & $9.1(8.0-10.3)$ & 213 & $15.8(13.6-17.9)$ & 44 & $3.0(2.1-3.9)$ & $5.2(3.8-7.2)$ \\
\hline Total & 3352 & $6.0(5.8-6.2)$ & 3040 & $11.0(10.6-11.4)$ & 312 & $1.1(1.0-1.2)$ & $9.9(8.8-11.1)$ \\
\hline
\end{tabular}

Personal non-commercial use only. The Journal of Rheumatology Copyright $\subset$ 2011 . All rights reserved. 
Table 3. Mortality rate (deaths per 1000 case person-years) and male/female rate ratio (M/F RR) of treated primary Sjögren's syndrome in Taiwan, 2005-2007.

\begin{tabular}{|c|c|c|c|c|c|c|c|}
\hline \multirow[b]{2}{*}{ Age, yrs } & \multicolumn{2}{|r|}{ Total } & \multicolumn{2}{|r|}{ Women } & \multicolumn{2}{|r|}{ Men } & \multirow[b]{2}{*}{$\mathrm{M} / \mathrm{F} \mathrm{RR}(95 \% \mathrm{CI}$} \\
\hline & No. & Rate $(95 \% \mathrm{CI})$ & No. & Rate $(95 \% \mathrm{CI})$ & No. & Rate $(95 \% \mathrm{CI})$ & \\
\hline $25-44$ & 7 & $6.1(1.6-10.6)$ & 5 & $4.7(0.6-8.7)$ & 2 & $24.7(-9.5-58.9)$ & $5.3(1.0-27.3)$ \\
\hline $65-74$ & 20 & $26.2(14.7-37.7)$ & 14 & $21.6(10.3-32.9)$ & 6 & $53.1(10.6-95.6)$ & $2.5(1.0-6.4)$ \\
\hline $75+$ & 22 & $59.4(34.6-84.2)$ & 16 & $52.9(27.0-78.8)$ & 6 & $88.2(17.6-158.8)$ & $1.7(0.7-4.3)$ \\
\hline Total & 64 & $13.4(10.2-16.7)$ & 49 & $11.4(8.2-14.6)$ & 15 & $33.4(16.5-50.4)$ & $2.9(1.7-5.3)$ \\
\hline
\end{tabular}

The second limitation was that the relevant clinical and laboratory data included in applications for a CIC for pSS were not electronically recorded in the catastrophic illness records of claims data and were therefore not accessible for examination, which rendered further analysis by symptoms and laboratory data unfeasible. The third limitation was that we did not calculate a prevalence rate because of relatively low numbers of patients applying for a CIC for pSS before 2005, which made the estimation of prevalence imprecise. A fourth limitation was that we could not calculate incidence rates by rural and urban areas because information on patient's place of residence was not available in claims data. Finally, a fifth limitation was the short followup period in calculating mortality and a lack of information regarding cause of death in the catastrophic illness records.

To our knowledge, this study identified the largest number of male incident cases (i.e., 312 patients) compared with previous studies, which could provide more accurate estimations of age-specific incidence in men. Consequently, we were able to compute the female/male ratio of incidence of pSS by age group, and found that the ratio was largest in the group aged 35-54 years, at around 14. In older adults, the female/male ratio decreased with age. The mean age at diagnosis of pSS was found to be 53 years for women in this study, which was older than the mean age of 51 years identified in a Slovenian study ${ }^{2}$ and younger than the 59 and 55 years reported in studies in the $\mathrm{US}^{1}$ and $\mathrm{Greece}^{3}$, respectively. We also found that the mean age of diagnosis of pSS in men was greater than that in women in Taiwan.

With regard to mortality, our findings were similar to those of previous studies ${ }^{3,4,5,6,7}$, i.e., mortality in patients with pSS was not significantly higher than that in the general population. Very few studies have examined the gender differences in mortality in patients with pSS. One Finnish study suggested that mortality in female patients with pSS is higher than that in males $^{5}$; however, we found that mortality in male patients with pSS was 3 times higher than that in female patients, and further study is needed to determine why this should be the case.

This nationwide population-based study was the first to use NHI claims data to estimate the incidence of treated
pSS. The large number of cases rendered estimation of sex- and age-specific incidence feasible. Generally, the incidence of treated pSS was found to be 10 times higher in women than in men, and 14 times higher in women than men in the group age $45-54$ years; however, mortality was 3 times higher in male patients with treated pSS than in female patients.

\section{REFERENCES}

1. Pillemer SR, Matteson EL, Jacobsson LTH, Martens PB, Melton LJ, O'Fallon WM, et al. Incidence of physician-diagnosed primary Sjogren's syndrome in residents of Olmsted County, Minnesota. Mayo Clin Proc 2001;76:593-9.

2. Plesivcnik Novljan M, Bozman B, Hocevar A, Grmek M, Kveder T, et al. Incidence of primary Sjogren's syndrome in Slovenia. Ann Rheum Dis 2004;63:874-6.

3. Alamanos Y, Tsifetaki N, Voulgari PV, Venetsanopoulou AI, Siozos C, Drosos AA. Epidemiology of primary Sjogren's syndrome in north-west Greece, 1982-2003. Rheumatology 2006;45:187-91.

4. Martens PB, Pillemer SR, Jacobsson LTH, O'Fallon WM, Matteson EL. Survivorship in a population based cohort of patients with Sjogren's syndrome, 1976-1992. J Rheumatol 1999;26:1296-300.

5. Pertovaara M, Pukkala E, Laippala P, Miettinen A, Pasternack A. A longitudinal cohort study of Finnish patients with primary Sjogren's syndrome: clinical, immunological, and epidemiological aspects. Ann Rheum Dis 2001;60:467-72.

6. Ioannidis JPA, Vassiliou VA, Moutsopoulos HM. Long-term risk of mortality and lymphoproliferative disease and predictive classification of primary Sjogren's syndrome. Arthritis Rheum 2002;46:741-7.

7. Theander E, Manthorpe R, Jacobsson LTH. Mortality and cause of death in primary Sjogren's syndrome. Arthritis Rheum 2004;50:1262-9.

8. Bureau of National Health Insurance. National health insurance profile 2010. [Internet. Accessed November 23, 2010.] Available from:

http://www.nhi.gov.tw/english/webdata.asp?menu=11\&menu_id=29 0\&webdata_id=2974

9. Bureau of National Health Insurance. Catastrophic illness patients. [Internet. Accessed November 23, 2010.] Available from: http://www.nhi.gov.tw/english/webdata.asp?menu=11\&menu_id=59 6\&webdata_id $=3180$

10. Vitali C, Bombardieri S, Jonsson R, Moutsopoulos HM, Alexander EL, Carsons SE, et al. Classification criteria for Sjogren's syndrome: a revised version of the European criteria proposed by the American-European Consensus Group. Ann Rheum Dis 2002;61:554-8. 